\title{
Multiextrapolação de Richardson com interpolação para reduzir o erro de discretização em malhas não uniformes 1D
}

\section{Carlos Alberto Rezende Carvalho Junior ${ }^{1}$}

Programa de Pós-Graduação em Métodos Numéricos em Engenharia (PPGMNE), Universidade Federal do Paraná (UFPR), Curitiba, PR

\section{Carlos Henrique Marchi ${ }^{2}$}

Departamento de Engenharia Mecânica, UFPR, Curitiba, PR

\section{Márcio André Martins ${ }^{3}$}

Departamento de Matemática, Universidade Estadual do Centro Oeste (UNICENTRO), Guarapuava, PR

\begin{abstract}
Resumo. Considera-se o emprego de Multiextrapolação de Richardson (MER) para reduzir o erro de discretização utilizando uma nova metodologia, proposta em 2013 para malhas uniformes, que envolve o emprego de interpolações polinomiais. Com a utilização dessa metodologia, MER teve seu desempenho melhorado: a magnitude dos erros de discretização reduziu progressivamente com o refinamento da malha, com um concomitante aumento das suas ordens de acurácia. Neste trabalho essa metodologia foi estendida para malhas não uniformes unidimensionais. Como problema-modelo considerou-se a equação de Poisson 1D, utilizando dois tipos de malha, e refinamento uniforme. A discretização dessa equação foi realizada com o método de diferenças finitas. Nas variáveis de interesse estudadas, foram alcançados resultados semelhantes com os apresentados para este mesmo problema com malhas uniformes, com MER, isto é, o erro de discretização teve redução significativa.
\end{abstract}

Palavras-chave. Erro de discretização, Multiextrapolação de Richardson (MER), Interpolação Polinomial, Método de diferenças finitas, Equação de Poisson 1D.

\section{Introdução}

Segundo [7], um dos grandes desafios encontrados em Dinâmica dos Fluidos Computacional (CFD) diz respeito ao nível de acurácia das soluções numéricas. Embora os erros numéricos não possam ser eliminados, eles devem ser minimizados. Dentre as fontes do erro numérico, o erro de discretização é o mais relevante.

\footnotetext{
${ }^{1}$ carloscarvalhoj@gmail.com

${ }^{2}$ marchi@ufpr.br

${ }^{3}$ mandre@unicentro.br
} 
A técnica denominada extrapolação de Richardson (ER) e sua versão aprimorada a multiextrapolação de Richardson (MER), teve início há muitos anos com [8,9], impulsionada pelo objetivo de se reduzir o erro de discretização $(E h)$. Desde seu surgimento, este método tem sido analisado, aprimorado ou simplesmente utilizado para a redução de $E h$, por diversos autores: $[3-7,11]$, entre outros.

O estímulo deste trabalho se deve aos resultados significativos de MER, na redução de Eh e aumento da ordem de acurácia (P), em condições ideais, com um custo computacional extremamente baixo. Entretanto, considerando-se malhas uniformes, em alguns casos relatados em [7], MER já não apresenta desempenho eficaz, sendo necessário uma metodologia alternativa para empregá-la com sucesso. Em [6] propõe-se o uso de MER precedida de interpolação polinomial, e, em alguns, também de métodos de otimização, tendo êxito nas variáveis de interesse estudadas, porém utilizou-se somente malhas uniformes. Neste trabalho, aplica-se este método em malhas não uniformes. Para isso, como problema teste, considera-se a equação de Poisson 1D e o método de diferenças finitas, com o estudo de diversas variáveis de interesse.

A continuidade do texto consiste da seguinte forma: na seção 2 é apresentado o modelo matemático utilizado, um resumo do método numérico empregado, a geração das malhas e a aplicação de MER, e na seção 3 são abordados os resultados e conclusão do trabalho.

\section{Metodologia}

\subsection{Problema modelo}

O modelo matemático considerado neste trabalho é uma variação da equação Poisson 1D com condições de contorno de Dirichlet:

$$
\frac{d T^{2}}{d x^{2}}=-\frac{9}{5} e^{3 x}, \quad T(0)=\frac{9}{5}, \quad T(1)=-\frac{e^{3}}{5}+3 .
$$

Em que $T$ representa a variável dependente (temperatura) e $x$ representa a variável independente (coordenada espacial). Como esta equação diferencial possui solução analítica, segundo $[4,10]$, somente neste caso é possível mensurar exatamente o erro numérico, condição essencial para o estudo realizado neste trabalho.

\subsection{Modelo numérico}

Para resolver numericamente a equação (1), utiliza-se o método de diferenças finitas (MDF) com aproximação do tipo CDS (Central Difference Scheme), para o caso da discretização não uniforme [4], com $\mathrm{P}=1$, sobre o domínio $\Omega[0,1]$. Com intuito de resolver o sistema linear tridiagonal gerado da discretização, utiliza-se o método TDMA (TriDiagonal Matrix Algorithm) [2].

\subsection{Geração das malhas}

Exitem várias formas de se refinar uma malha não uniforme, como pode ser visto em $[4,10]$. Neste trabalho utiliza-se refino uniforme com razão dois. Com o emprego 
de refino uniforme, segundo [10], em geral, $\mathrm{P}$ tende ao seu valor teórico, obtido a priori. Considera-se como base para os refinos a malha inicial (Figura 1), onde as constantes $h_{i}$, para $i=1,2, \ldots, n$, são os espaçamentos positivos não nulos entre os nós, de modo que

$$
\sum_{i=1}^{n} h_{i}=L
$$

Os pontos $P_{i}$, para $i=1,2, . ., n-1$, correspondem aos pontos nodais da malha inicial, com exceção dos extremos e a constante $L$, corresponde ao comprimento do intervalo. Considerase $L=1$. As entradas $h_{i}$ são fornecidas, respeitando os critérios comentados anteriormente.

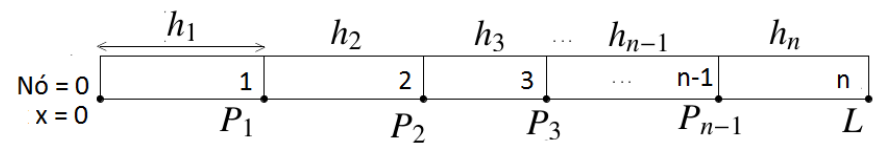

Figura 1: Malha inicial $\left(\Omega^{h_{1}}\right)$, sobre o intervalo $[0, L]$.

\subsection{Aplicação de MER}

Para aplicar MER, considera-se a obtenção da solução numérica de uma determinada variável de interesse $(\phi)$, em $G$ malhas distintas $\Omega^{h^{1}}$ (malha inicial), $\Omega^{h^{2}}, \ldots, \Omega^{h^{G}}$, geradas com razão de refino $r=h_{g-1} / h_{g}=2(g=2, . ., G)$. O emprego de MER com $m$ níveis de extrapolações é dado por $[6,7]$ :

$$
\phi_{g, m}=\phi_{g, m-1}+\frac{\phi_{g, m-1}-\phi_{g-1, m-1}}{r^{p_{m-1}}-1} ; g=2, . ., G ; m=1, \ldots, g-1 .
$$

Segundo [6,7], para empregar MER visando uma redução efetiva em $E h$ associado à determinada variável de interesse $(\phi)$, em que se classifica $\phi$ em cinco tipos de acordo com a sua localização em malhas distintas, estabelecendo formas de obter a variável em cada caso, para em seguida aplicar MER. Neste trabalho considera-se três tipos de $\phi$, das cinco apresentadas por [6,7]: Tipo I são as variáveis $\phi$ locais, cuja localização $c$ é mantida em todas as malhas $(g)$ consideradas e coincide com um ponto nodal, ou ainda, é caracterizada por uma variável global; Tipo III considera variáveis $\phi$ locais em que $c$ não coincide com um ponto nodal de $g$, nem com o ponto médio entre coordenadas nodais, entretanto, possui, uma localização fixa; Tipo $\mathbf{V}$ são variáveis $\phi$ locais onde $c$ é desconhecida previamente, isto é, são variáveis que podem apresentar mudança de coordenada em malhas distintas. Obtém-se $\phi$ de acordo com as recomendações [6,7]: para o Tipo I, obtém-se $\phi$ em cada malha e emprega-se MER diretamente com a equação (3); para o Tipo III empregase interpolação polinomial, com grau máximo possível, para obtenção de $\phi$, em seguida considera-se a equação (3); para o Tipo V emprega-se interpolação polinomial com o grau máximo possível e, busca-se o seu ponto ótimo (máximo ou mínimo) para a determinação de $\phi$ em cada malha, e ao final considera-se a equação (3). 


\section{Resultados e conclusão}

As simulações foram realizadas utilizando precisão quádrupla em Fortran (linguagem utilizada). Considerou-se dois casos envolvendo malhas não uniformes. O primeiro (caso A), utiliza-se a malha inicial A (Figura 2) e obtêm-se as outras malhas a partir do processo de refino uniforme de razão dois. O segundo (caso B), utiliza-se a malha inicial B com 11 nós, cujos pontos $P_{i}$ são dados pela equação (4) e repete-se o processo de refino, utilizado no caso A.

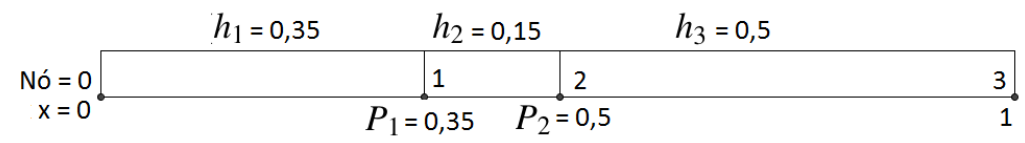

Figura 2: Malha inicial A.

Não houve critério na escolha dos pontos da malha A. Em contrapartida na malha B, através de um programa, obtêm-se os pontos $P_{i}$ de forma aleatória:

$$
\begin{aligned}
{\left[P_{1} ; P_{2} ; P_{3} ; P_{5} ; P_{6} ; P_{7} ; P_{8} ; P_{9}\right]^{T} \approx } & {[6,84 E-08 ; 4,45 E-03 ; 6,15 E-02 ; 1,16 E-01} \\
& 1,46 E-01 ; 5,85 E-02 ; 1,59 E-01 ; 1,45 E-01]^{T}
\end{aligned}
$$

Nas simulações feitas no caso A, foram utilizadas 25 malhas, cuja mais fina contém 50.331.649 nós. No caso B, foram 23 malhas, cuja malha mais fina contém 41.934 .041 nós. A variável Em significa erro de discretização (Eh) com MER, Ep e Epm significam erro de discretização sem e com MER com interpolação de ordem (grau) $p$. Neste trabalho apresentam-se apenas alguns resultados de $E p$, porque os resultados obtidos são similares e não sofrem muita alteração com o grau $p$ utilizado, exceto nas ordens maiores que apresentam rendimento inferior, nas malhas mais finas.

Para as variáveis do tipo I, analisaram-se várias $\phi$, entre elas temos a temperatura avaliada no ponto nodal $P_{1}$, representada por $T_{P_{1}}$. Para estas variáveis aplicou-se MER diretamente, através da equação (3) (obtém-se $E m$ ). Considera-se como variável do tipo III a temperatura avaliada no ponto $2 / 3$, representada por $T_{2 / 3}$. Para obtê-la, utilizou-se interpolação polinomial de ordens 1 a 10, em seguida avaliou-se o ponto $2 / 3$ em cada polinômio, e ao final, considerou-se o emprego da equação (3) (obtém-se Epm). Por fim, a temperatura máxima e o ponto onde a temperatura é máxima, representada por $T_{\max }$ e $x_{\max }$ respectivamente, são variáveis do tipo $\mathbf{V}$, para obtê-las utilizou-se interpolação polinomial de ordens 2 a 10. Em seguida considerou-se a busca do seu ponto de máximo pelo método iterativo de Newton; e ao final, considerou-se o emprego da equação (3) (obtém-se Epm).

Analisou-se o desempenho de MER nos dois casos (A e B). Entretanto devido à limitação de espaço, nesta seção são apresentados apenas os resultados do primeiro caso. Os resultados do caso B são similares e até melhores, para variáveis do tipo $\mathrm{V}$, devido ao fato da malha B possuir mais pontos, MER tem desempenho melhorado neste caso, resultado já esperado de [5]. As interpolações de ordens 9 e 10 são as que possuem maior 
custo computacional, e são consideradas como as interpolações de grau máximo possível, sugeridos pela metodologia.

As abscissas $(h)$ dos gráficos são feitas com a média necessária para o espaçamento $h$ entre os nós para discretização, isto é este $h$ é o mesmo obtido para o caso da discretização em diferenças finitas, para o caso uniforme. Para variáveis do Tipo I, foram obtidos resultados excelentes com poucas extrapolações, como pode ser visto na Figura 3. A ordem de acurácia $(\mathrm{P})$ foi elevada para $\mathrm{P}>10$, nas variáveis analisadas. A variável $T_{2 / 3}$ (Tipo III) apresentou melhores resultados Epm com graus 6, 7, 8, 9 e 10 (Figura 3). A ordem de acurácia foi elevada para $\mathrm{P}>8 \mathrm{com}$ a utilização desses polinômios. Na variável $x_{\max }$ (Tipo V), Epm foi influenciado pela falta de pontos nas malhas iniciais, como pode ser visto na figura 4 , fato que não influenciou a obtenção da variável $T_{\max }$ (Tipo V) (Figura 5). Os melhores resultados da variável Epm foram com os graus 7, 8 e 9 nesta malha, os graus 6 e 10 tiverem desempenho levemente inferior devido ao problema relatado anteriormente. Porém na malha B, obtém-se os melhores resultados com os graus $6,7,8$, 9 e 10. As ordens de acurácia com estes polinômios foram maiores que dez. De acordo com os resultados obtidos conclui-se que a metodologia empregada através da obtenção das variáveis Epm e Em melhoram o desempenho de MER, e consequentemente obtemos significativa redução de $E h$, também para malhas não uniformes unidimensionais, nas variáveis analisadas (Tipo I, III e V). Segundo [7] este procedimento é caracterizado como um pós-processamento, e apresenta baixíssimo custo computacional. Para variáveis do tipo III, em relação ao custo computacional, sugere-se a utilização da interpolação de ordem 6 e para o tipo V sugere-se a interpolação de ordem 8, pois resultaram em comportamento semelhante e até melhores, no caso da interpolação de grau 8 em comparação com a de grau 10 na variável $x_{\max }$.

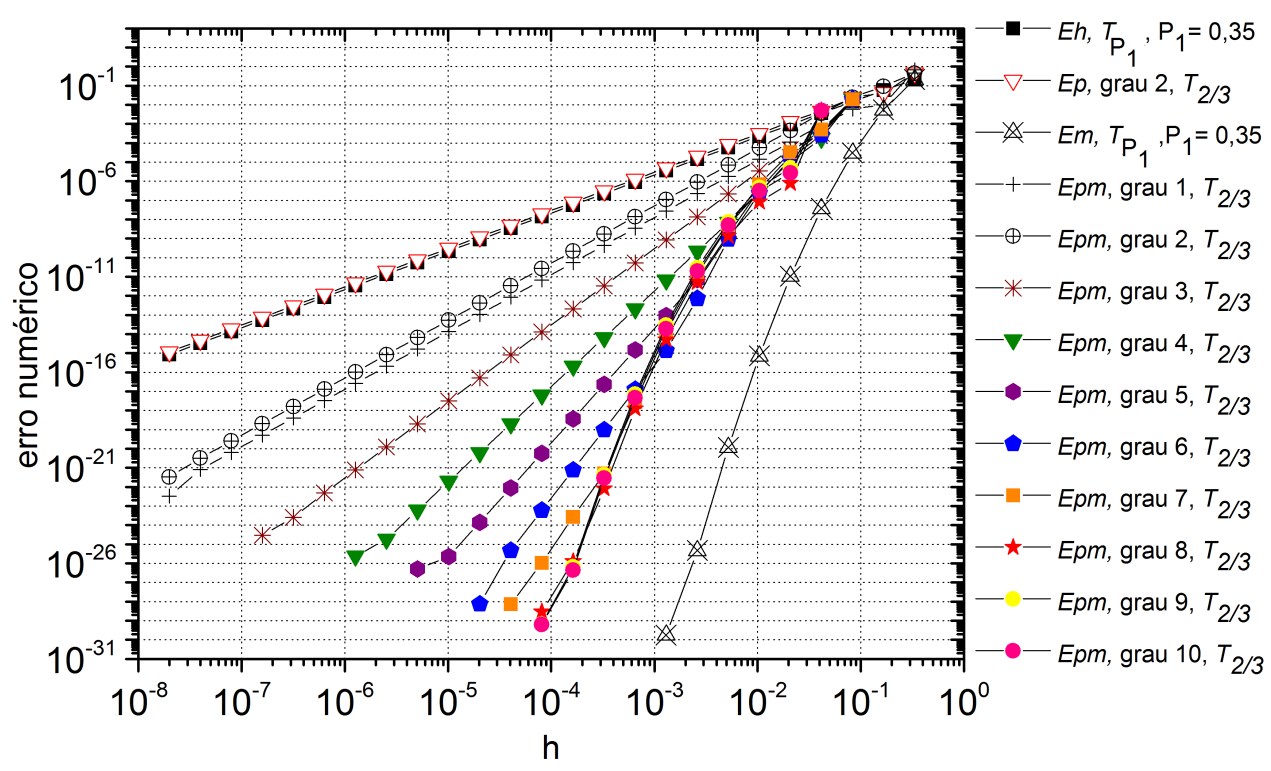

Figura 3: Eh, Ep, Em e Epm para variável do Tipo I e III, caso A. 


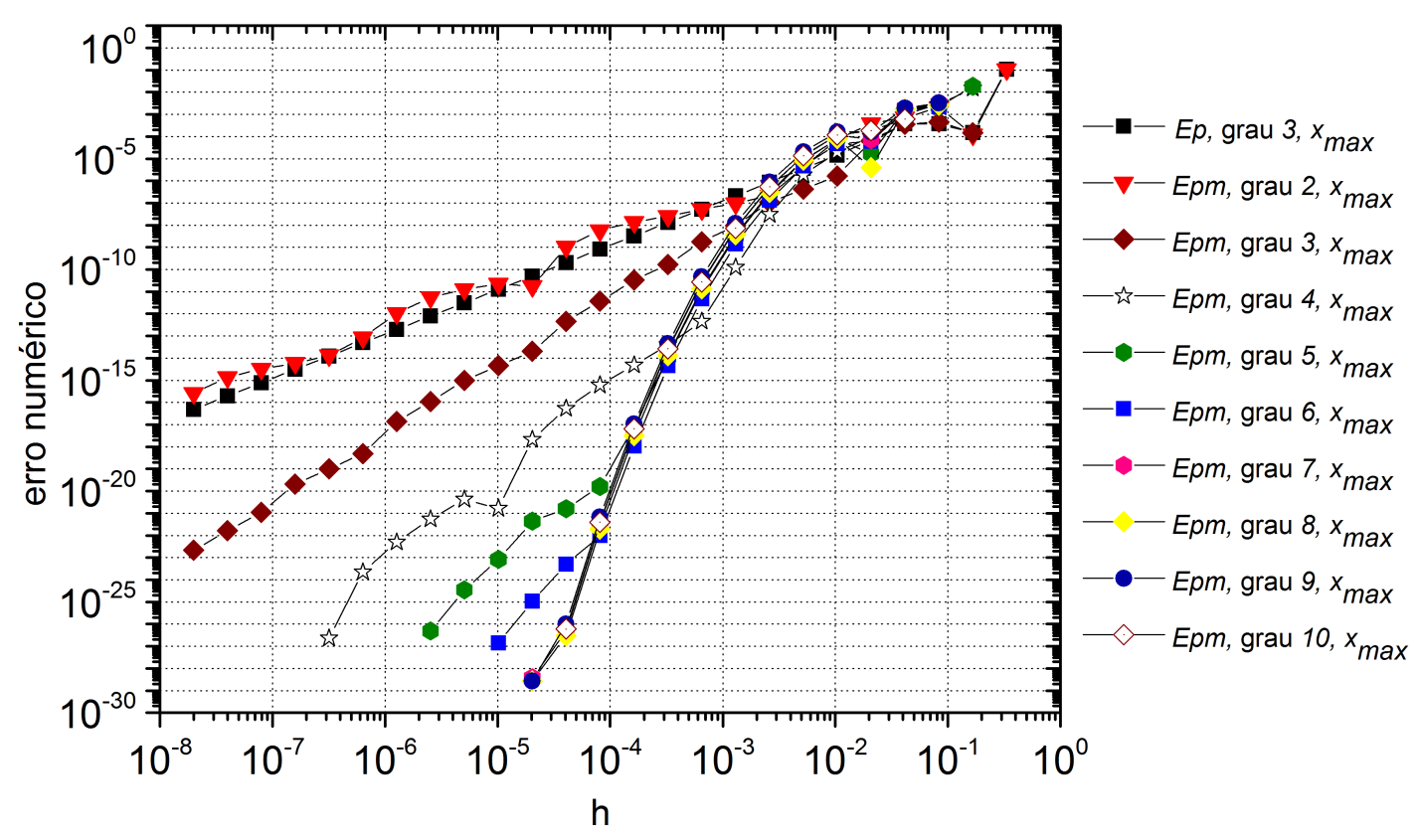

Figura 4: Ep e Epm para variável $x_{\max }$, caso A.

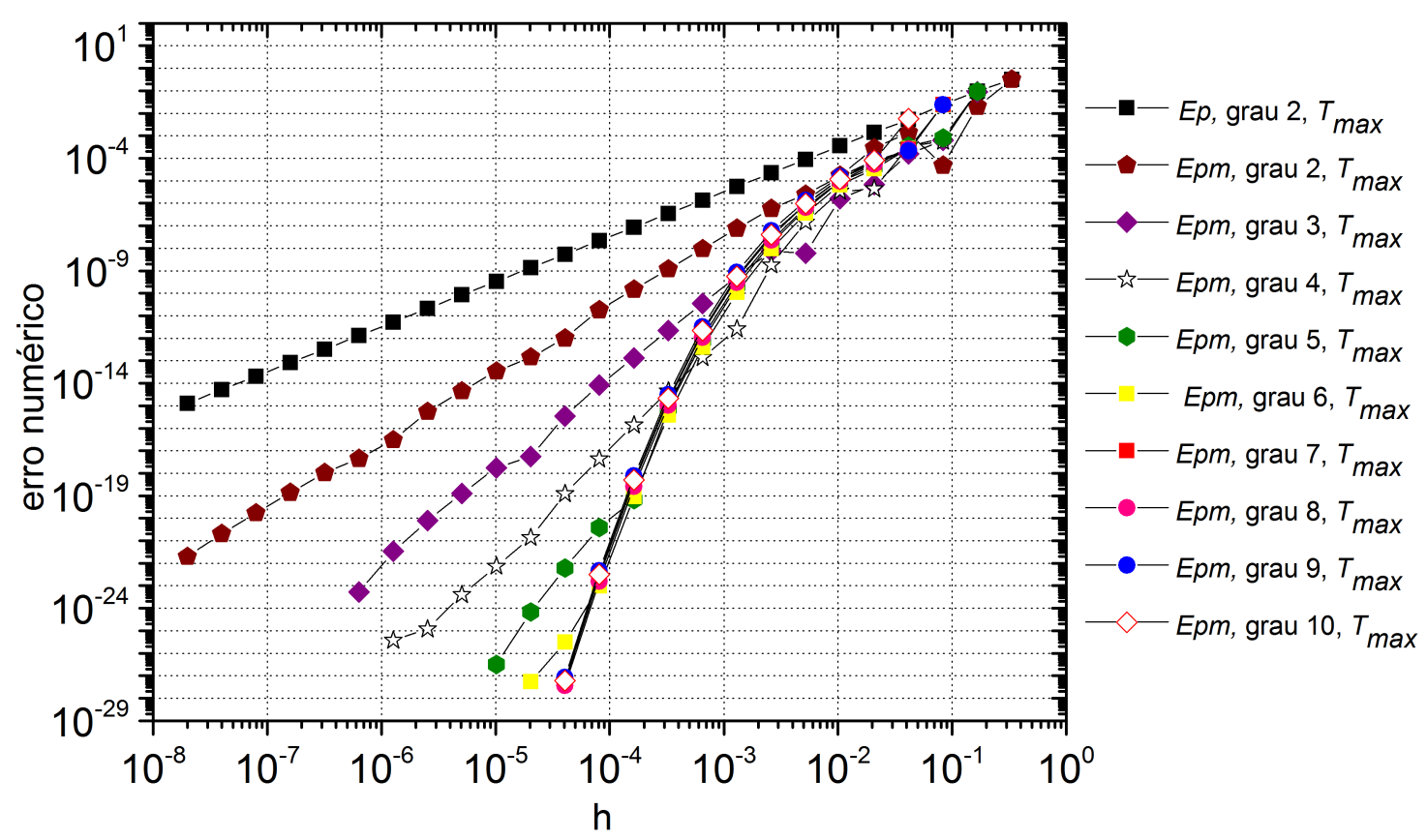

Figura 5: $E p$ e $E p m$ para variável $T_{m a x}$, caso A. 


\section{Agradecimentos}

Os autores agradecem ao apoio financeiro da CAPES (Coordenação de Aperfeiçoamento de Pessoal e Nível Superior) e CNPq (Conselho Nacional de Desenvolvimento Científico e Tecnológico). O primeiro autor agradece ao PPGMNE. O segundo autor é bolsista do CNPq. O terceiro autor agradece a UNICENTRO.

\section{Referências}

[1] C.A. Alves, Verificação de soluções numéricas da equação de Laplace 2D com malhas triangulares e múltiplas extrapolações de Richardson, Tese de Doutorado em Engenharia Mecânica, UFPR, (2010).

[2] A. Fortuna, Técnicas computacionais para dinâmica de fluidos, EDUSP, (2000).

[3] F. F. Giacomini e C.H. Marchi, Efeito da razão de refino de malha sobre o uso de multiextrapolações de Richardson em CFD, XXXV Congresso Nacional de Matemática Computacional, (2014).

[4] C.H. Marchi, Verificação de soluções numéricas unidimensionais em dinâmica de fluidos, Tese de Doutorado em Engenharia Mecânica, UFSC, (2001).

[5] C.H. Marchi e E.M. Germer, Verificação de esquemas advectivo-difusivos 1D com e sem múltiplas extrapolações de Richardson, Proceeding of the XXX Iberan Latin American Congress on the Computational Methods in Engineering, (2009).

[6] M.A. Martins, Multiextrapolação de Richardson com interpolação para reduzir e estimar o erro em CFD, Tese de Doutorado em Engenharia Mecânica, UFPR, (2013).

[7] M. A. Martins, C.H. Marchi, L.A. Novak, M.A.V. Pinto, L.K. Araki e S.F.T. Gonçalves, Multiextrapolação de Richardson com interpolação para reduzir o erro de discretização em CFD, Congresso de Matemática Aplicada e Computacional, (2013).

[8] L.F. Richardson, The approximate arithmetical solution by finite diferences of physical problems involving differential equation, with an application to the stresses in a masonry dam, Phylosophical Proceedings of the Royal Society of London, v.210, Serial A, p. 307-357, (1910).

[9] L.F. Richardson and J.A. Gaunt, The differed approach to the limit, Phylosophical Proceedings of the Royal Society of London, v. 226, Serial A, p. 229-361, (1927).

[10] F.A. Schneider, Verificação de soluções numéricas em problemas difusivos e advectivos com malhas não uniformes, Tese de Doutorado em Métodos Numéricos em Engenharia, UFPR, (2007).

[11] A.P. Vargas, Multiextrapolação de Richardson e esquemas de $1^{\circ}$ e $2^{\circ}$ ordens, mistos e Crank-Nicolson sobre as equações 2D de Advecção-Difusão e Fourier, Tese de Doutorado em Engenharia Mecânica, UFPR, (2013). 